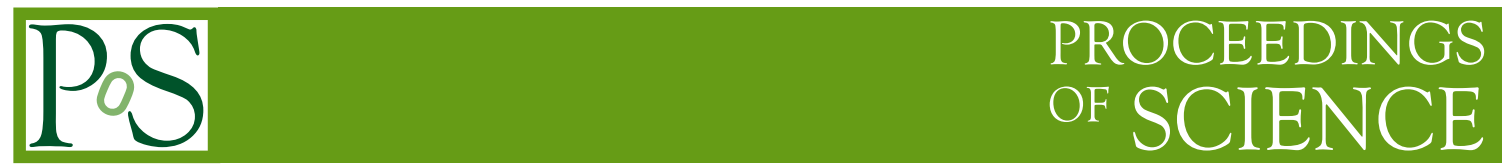

\title{
Photon-jet production cross section and angular correlations in high energy proton-nucleus collisions
}

\section{Jamal Jalilian-Marian*}

Natural Sciences Department, Baruch College, 17 Lexington Ave., New York, NY, USA

E-mail: jamal.jalilian-marian@baruch.cuny.edu

We calculate the differential cross section for production of a photon-jet (quark) pair in high energy proton-nucleus collisions where we include contributions of both small and large $x$ gluons of a target nucleus. This generalizes the Color Glass Condensate-based calculations and allows one to investigate the production cross section and the photon-jet angular correlations at both low and high transverse momentum in both forward and mid-rapidity regions.

HardProbes 2020

1-6 June 2020

Austin, Texas

${ }^{*}$ Speaker 


\section{Introduction}

Color Glass Condensate (CGC) is an effective theory of QCD at high energy, or equivalently at small $x$ [1]. It describes the dynamics of gluon saturation in a proton or a nucleus at high energy where the proton or nucleus is a dense system of small $x$ gluons carrying a very small fraction $(x)$ of the hadron energy. To probe this dense system of gluons in a hadron or nucleus various observables such as structure functions, single and double inclusive particle production in Deep Inelastic Scattering (DIS) experiments, as well as particle production in proton-nucleus (dilute-dense) collisions have been investigated.

Due to the high density of the gluons in the target proton or nucleus one can not use the collinear factorization approach of perturbative QCD which is appropriate when the proton or nucleus is a dilute system of partons. A more appropriate formalism for particle production in the forward rapidity region of a proton-nucleus collision is the so called hybrid formalism [2] where the projectile proton is treated as a dilute system of partons while the target nucleus is treated as a dense system of gluons. This is due to the kinematic relation between the transverse momentum $p_{t}$ and rapidity $y$ of the produced hadron with the Bjorken $x_{p, t}$ of the partons in the projectile and target given by

$$
x_{p, t}=\frac{p_{t}}{\sqrt{s}} e^{ \pm y}
$$

which shows that one probes the large $x(\sim 1)$ partons of the projectile and small $x(\ll 1)$ partons of the target when a particle is produced in the forward rapidity region. The hybrid formulation of particle production in the CGC formalism is now extended to Next to Leading Order (NLO) accuracy, including resummation of various kinematic factors that are important in the particular kinematics limits. Despite all the progress made in this direction, the applicability of CGC formalism is still limited to the small $x$ region, commonly taken to be $x<0.01$. In a proton-nucleus type collision this limits the the range of transverse momentum where the CGC formalism can be applied. Furthermore, by making the small $x$ approximation one may be drastically overestimating the significance of small $x$ kinematics since, unlike the collinear factorization formalism, recoil is neglected and the underlying partonic process is effectively a $2 \rightarrow 1$ process [3]. To remedy this it is essential to generalize the CGC formalism to include large $x$ partons of the target. In [4] we proposed a new formalism which includes scattering from both small and large $x$ partons of the target.

\section{Scattering from both small and large $x$ gluons of the target}

Inclusion of multiple soft scatterings of a projectile parton from small $x$ gluons of a target hadron can be resummed into a Wilson line; a path ordered exponential of the gluon field representing the target hadron or nucleus. This requires the so called eikonal approximation where the projectile parton exchanges small transverse momenta with the target and as a result stays on a straight line, i.e., no large angle deflection. Due to this approximation, the resulting degrees of freedom describing the dense gluon system in the target hadron are correlation functions of Wilson lines [5], with the two-point function (called a dipole) the most common one. The physical observables are then given in terms of correlations of Wilson lines which in turn satisfy the JIMWLK evolution equation [1] 
governing their energy (or $x$ ) dependence. Due to the eikonal approximation inherent to CGC calculations at the classical level, the scattered partons can not undergo large angle deflections. In other words the transverse momentum of the scattered parton must be much smaller than the energy of the incoming parton. This limits the regime of validity of CGC calculations to low $p_{t}$ observables [6]. Therefore it is essential to go beyond eikonal approximation and to include large angle scattering. To do this we must include scattering from large $x$ partons of the target which can result in exchange of longitudinal as well as large transverse momentum between the projectile and the target causing a large angle deflection of the scattered parton.

In [4] we calculated the cross section for scattering of a quark from both small and large $x$ gluons of the target. Specifically, we considered scattering of a projectile parton of a given helicity [7] on the unpolarized target and showed that inclusion of large parton of the target leads to a spin asymmetry in the production cross section. The amplitude (for brevity only the Dirac numerators are shown) for scattering of a positive helicity quark is given by

$$
\mathcal{N}_{a}^{+}=\mathcal{N}_{1, a}^{+}+\mathcal{N}_{2, a}^{+}+\mathcal{N}_{3, a}^{+}
$$

with

$$
\begin{aligned}
\mathcal{N}_{1, a}^{+} & =p^{+} \sqrt{\frac{q^{+}}{p^{+}}}\left\{2 A_{a}^{-}(x)-A_{a}^{i}(x)\left[\frac{k_{1 i}-i \epsilon_{i j} k_{1}^{j}}{q^{+}}+\frac{k_{i}+i \epsilon_{i j} k^{j}}{p^{+}}\right]\right\} \\
\mathcal{N}_{2, a}^{+} & =\frac{p^{+}}{q_{\perp}^{2}} \sqrt{\frac{q^{+}}{p^{+}}}\left\{\left(1+\frac{q^{+}}{p^{+}}\right) q_{\perp} \cdot A_{\perp}^{b}(x)+i\left(1-\frac{q^{+}}{p^{+}}\right) \epsilon^{i j} q_{i} A_{j}^{a}(x)\right\} \\
\mathcal{N}_{3, a}^{+} & =\mathcal{N}_{2, a}^{+}\left(q_{i} \rightarrow p_{1 i}\right) \\
& =\frac{p^{+}}{p_{1 \perp}^{2}} \sqrt{\frac{q^{+}}{p^{+}}}\left\{\left(1+\frac{q^{+}}{p^{+}}\right) p_{1 \perp} \cdot A_{\perp}^{b}(x)+i\left(1-\frac{q^{+}}{p^{+}}\right) \epsilon^{i j} p_{1 i} A_{j}^{a}(x)\right\}
\end{aligned}
$$

and the negative helicity amplitudes are related to the positive helicity ones by

$$
\mathcal{N}_{1,2,3}^{-a}=\left[\mathcal{N}_{1,2,3}^{+a}\right]^{\star}
$$

where $p, q$ are the momenta of the incoming and outgoing quark respectively, $k$ and $k_{1}$ are the momenta of the internal quark line before and after the hard scattering which is integrated over while $a$ is a color index. The three sub-amplitudes correspond to the cases when only the initial and final state quarks undergo multiple soft scattering, when only the large $x$ gluon undergoes multiple soft scatterings, and when only the final state quark and the large $x$ gluon undergo multiple soft scatterings. These expressions can be used to investigate baryon transport as well as high $p_{t}$ spin asymmetries and collective phenomena in proton-nucleus collisions. The next step would be to calculate the one-loop corrections to this cross section which would include radiation of gluons (as part of the real corrections). To do this one would need to consider radiation of a gluon from the initial and final state quarks, before or after a hard scattering as well as radiation from the large $x$ gluon. Doing this (and including the virtual corrections) would lead to a scattering cross section which would include both the collinear factorization limit in the high $p_{t}$ region and the CGC hybrid factorization in the low $p_{t}$ (small $x$ ) region. Inclusion of gluon radiation above would not only allow one to generalize the CGC cross sections to include large $x$ effects in single inclusive hadron 
(jet) production, it will also allow one to investigate di-hadron (di-jet) production and angular correlations at both low and high $p_{t}$, thus generalizing the CGC results which are valid at low $p_{t}$ and unifying it with the more common collinear factorization formalism.

There is a simpler process which exhibits many features of di-hadron correlations, namely, photon-hadron (jet) production cross section and angular correlations. This is simpler than gluon radiation due to two reasons; photons do not interact with the background field, and the large $x$ gluon can not radiate a photon which will simplify the calculations tremendously. Therefore we will concentrate on the photon radiation here as a first step toward calculation of gluon radiation. As we are going beyond eikonal approximation there will be contributions which would be suppressed in the eikonal limit but will need to be included here. For example, radiation before and after multiple soft scatterings is suppressed by powers of energy $\mathrm{n}$ the eikonal limit but not at large $x$. Furthermore one will need to include radiation by a parton while inside the target, i.e., one needs to go beyond the shock wave limit and include the finite width of the target.

There are two classes of diagrams in general corresponding to the cases whether the photon radiation happens before or after hard scattering. To be specific we will illustrate the case when the radiation happens after the hard scattering and focus on the case when the initial and final state quarks (but not the large $x$ gluon) undergo multiple soft scatterings. The one needs to include any and all soft scatterings of the quark line. Part of this contribution to the scattering amplitude which exhibits all the important aspects of the result can be written as

$$
\begin{aligned}
i \mathcal{A}= & i \text { eg } \int \frac{d^{2} k_{1 t}}{(2 \pi)^{2}} \frac{d^{2} \bar{k}_{1 t}}{(2 \pi)^{2}} d^{4} r d^{2} r_{1 t} d^{2} \bar{r}_{1 t} d \bar{z}^{+} \\
& e^{i\left(\bar{q}^{+}+\bar{k}^{+}-p^{+}\right) r^{-}} e^{-i\left(\bar{k}_{1 t}-k_{1 t}\right) \cdot r_{t}} e^{i\left(\bar{q}^{-}+\bar{k}^{-}\right) \bar{z}^{+}} e^{-i\left(k_{1 t}-p_{t}\right) \cdot r_{1 t}} e^{-i\left(\bar{q}_{t}+\bar{k}_{t}-\bar{k}_{1 t}\right) \cdot \bar{r}_{1 t}} \\
& \bar{u}(\bar{q}) \notin(\bar{k}, \lambda) \frac{\Phi+\not k}{2\left(\bar{q}^{+}+\bar{k}^{+}\right)} \hbar \bar{V}\left(\bar{r}_{1 t} ; r^{+}, \bar{z}^{+}\right) \frac{\mathbb{k}_{1}}{2\left(\bar{q}^{+}+\bar{k}^{+}\right)} A(r) \frac{k_{1}}{2 p^{+}} \not V\left(r_{1 t} ;-\infty, r^{+}\right) u(p)
\end{aligned}
$$

where all the bar-ed quantities are in the frame where the final-state quark (after scattering from the large $x$ field $A$ ) has only a large + component of momentum. The semi-infinite and finite Wilson lines encode all the soft scatterings of the quark from the small $x$ gluons of the target while the field $A(r)$ is the color field radiated by large $x$ gluons of the target. Furthermore, $p$ is the momentum of the incoming quark while $\bar{q}, \bar{k}$ are the momenta of the final state quark and photon and $\notin$ is he polarization vector of the outgoing photon. Due to lack of space we do include the other contributions to the amplitude here.

The amplitude constructed can be used to calculate the production cross section for a pair of a photon and a quark which can then be used to investigate the azimuthal angular correlations between the photon and the quark (hadron or jet) in proton-nucleus collisions in the transverse momenta region where the standard CGC formalism is not valid. Furthermore, it will help clarify the analytic structure of the one-loop corrections when a gluon, rather than a photon is radiated, which will then be used to derive a production cross section both in high $p_{t}$ and at low $x$.

\subsection{Acknowledgments}

We acknowledge support by the DOE Office of Nuclear Physics through Grant No. DE-FG0209ER41620 and by PSC-CUNY through grant No. 62185-0050. 


\section{References}

[1] J. Jalilian-Marian and Y. V. Kovchegov, Prog. Part. Nucl. Phys. 56, 104-231 (2006) doi:10.1016/j.ppnp.2005.07.002 [arXiv:hep-ph/0505052 [hep-ph]].

[2] A. Dumitru and J. Jalilian-Marian, Phys. Rev. Lett. 89, 022301 (2002) doi:10.1103/PhysRevLett.89.022301 [arXiv:hep-ph/0204028 [hep-ph]], Phys. Lett. B 547, 15-20 (2002) doi:10.1016/S0370-2693(02)02709-0 [arXiv:hep-ph/0111357 [hepph]]; J. Jalilian-Marian and Y. V. Kovchegov, Phys. Rev. D 70, 114017 (2004) doi:10.1103/PhysRevD.71.079901 [arXiv:hep-ph/0405266 [hep-ph]].

[3] A. Dumitru, A. Hayashigaki and J. Jalilian-Marian, Nucl. Phys. A 765, 464-482 (2006) doi:10.1016/j.nuclphysa.2005.11.014 [arXiv:hep-ph/0506308 [hep-ph]]. V. Guzey, M. Strikman and W. Vogelsang, Phys. Lett. B 603, 173-183 (2004) doi:10.1016/j.physletb.2004.10.033 [arXiv:hep-ph/0407201 [hep-ph]].

[4] J. Jalilian-Marian, Phys. Rev. D 102, no.1, 014008 (2020) doi:10.1103/PhysRevD.102.014008 [arXiv:1912.08878 [hep-ph]], Phys. Rev. D 99, no.1, 014043 (2019) doi:10.1103/PhysRevD.99.014043 [arXiv:1809.04625 [hep-ph]], Phys. Rev. D 96, no.7, 074020 (2017) doi:10.1103/PhysRevD.96.074020 [arXiv:1708.07533 [hep-ph]].

[5] A. Dumitru and J. Jalilian-Marian, Phys. Rev. D 82, 074023 (2010) doi:10.1103/PhysRevD.82.074023 [arXiv:1008.0480 [hep-ph]]; A. Dumitru, J. JalilianMarian and E. Petreska, Phys. Rev. D 84, 014018 (2011) doi:10.1103/PhysRevD.84.014018 [arXiv:1105.4155 [hep-ph]].

[6] E. M. Henley and J. Jalilian-Marian, Phys. Rev. D 73, 094004 (2006) doi:10.1103/PhysRevD.73.094004 [arXiv:hep-ph/0512220 [hep-ph]]; F. Gelis and J. Jalilian-Marian, Phys. Rev. D 67, 074019 (2003) doi:10.1103/PhysRevD.67.074019 [arXiv:hep-ph/0211363 [hep-ph]], Phys. Rev. D 66, $094014 \quad$ (2002) doi:10.1103/PhysRevD.66.094014 [arXiv:hep-ph/0208141 [hep-ph]], Phys. Rev. D 76, 074015 (2007) doi:10.1103/PhysRevD.76.074015 [arXiv:hep-ph/0609066 [hep-ph]]; J. Jalilian-Marian, Nucl. Phys. A 753, 307-315 (2005) doi:10.1016/j.nuclphysa.2005.02.156 [arXiv:hep-ph/0501222 [hep-ph]], Nucl. Phys. A 739, 319-328 (2004) doi:10.1016/j.nuclphysa.2004.04.103 [arXiv:nucl-th/0402014 [nucl-th]], Phys. Rev. C 70, 027902 (2004) doi:10.1103/PhysRevC.70.027902 [arXiv:nucl-th/0212018 [nuclth]]; J. Jalilian-Marian, S. Jeon and R. Venugopalan, Phys. Rev. D 63, 036004 (2001) doi:10.1103/PhysRevD.63.036004 [arXiv:hep-ph/0003070 [hep-ph]].

[7] A. Ayala, M. Hentschinski, J. Jalilian-Marian and M. E. Tejeda-Yeomans, Nucl. Phys. B 920, 232-255 (2017) doi:10.1016/j.nuclphysb.2017.03.028 [arXiv:1701.07143 [hep-ph]], Phys. Lett. B 761, 229-233 (2016) doi:10.1016/j.physletb.2016.08.035 [arXiv:1604.08526 [hep$\mathrm{ph}]]$. 